\title{
Helioseismic inferences
}

\author{
Hiromoto Shibahashi \\ Department of Astronomy, University of Tokyo, Tokyo 113-0033, Japan \\ email: shibahashi@astron.s.u-tokyo.ac.jp
}

\begin{abstract}
The brilliant outcome of some 30 years of helioseismology spreads over a wide range of topics. Some highlights relevant to the cause of the solar activity cycle are listed up. The rotation profile in the solar convective zone is discussed as an important source of the dynamo mechanism. The kinematic dynamo model is described in the linear approximation, and the condition for the solar type dynamo is derived. It is shown that comparison of this condition with the rotation profile determined from helioseismology is useful to identify the possible seats of the dynamo.
\end{abstract}

Keywords. magneothydrodynamics: MHD, Sun: activity, Sun: helioseismology, Sun: magnetic fields

\section{Introduction}

It is almost half a century ago that the solar surface was found by Leighton et al. (1962) to be almost entirely covered with vertically oscillating elements. As observations were accumulated and they were compared in detail with various theoretically proposed interpretations, it became clear by the late 1970s that these oscillations are superposition of nonradial p-modes of the sun with degrees $l \simeq 200-1000$. Observations of the sun using the integrated sunlight over the whole disk, which were first performed by the Birmingham group also in the late 1970s, clarified that the sun is also oscillating with pmodes with low degrees $l \simeq 0-4$. The gap between low $l$ and high $l$ p-modes in detection of solar oscillations was later filled, and it became eventually clear that the sun is pulsating with millions of its eigenmodes. The detection of the eigenmodes of the sun has opened a new field of research called helioseismology, providing a new tool to see the invisible interior of the sun. Ground-based network observations (BiSON, GONG, IRIS, and TON) and uniterrupted observations from space (SOHO) developed significantly helioseismolgy, and various helioseismic methods are now providing new insight into understanding of the solar acitivity, which is one of the most important issues in solar physics.

\section{Outcome of some 30 years of helioseismology}

The outcome of some 30 years of helioseismology is brilliant and spreads over a wide range of topics. Listed below are only some highlights.

- Finding of the fact that the sun is oscillating with a lot of its eigenmodes, - that is, the sun is now regarded as a pulsating star while it had never been thought so before.

- The recent observations of solar oscillations provide us with accurate frequencies of a large number of solar eigenmodes. The relative errors in some of the measured frequencies are as small as $10^{-6}$.

- Determination of the sound-speed and density profiles in the sun: By the inversion of these frequencies, we can determine functional forms of the acoustic quantities, the sound speed profile and the density profile, as a function of radius respectively. We now 
know the sound speed profile within the errors of a few tenths of a percent, and the density profile with the order of $1 \%$ errors, in the range of $0.2 \lesssim r / R_{\odot} \lesssim 0.95$.

- Calibration of the depth of the convection zone: Convection is still one of the poorly understood physics in making theoretical models of stars. However, the extent of the convection zone in the sun is now accurately determined from the sound-speed profile determined helioseismically .

- Finding of a new mechanism of driving eigenoscillations, - that is, turbulent convection in a star generates acoustic noise propagating inside the star, and the waves resonating in the star, which is regarded as a wave cavity, survive and are maintained at some level.

- Based on the discovery of very small amplitude pulsations found from observations of the sun as a star, we now expect that pulsations, at least with small amplitudes, will be ubiquitous among stars in general. This opens the possibility of a seismological approach to stars, which is now called "asteroseismology."

- Calibration of "standard evolutionary models": The sound-speed profile determined from helioseismology matches fairly well up to that of solar models constructed with standard evolution theory of stars, though such beautiful matching might be broken if the recently determined chemical abundances of the sun.

- Inference of helium abundance: A lack of appropriate spectroscopic lines of helium makes it hard to determine spectroscopically abundance of helium in the solar atmosphere. Helioseismology provides new methods of determination of helium abundance.

- Proof of importance of chemical diffusion and gravitational settling: Theoretical models of the sun constructed taking account of chemical diffusion and gravitational settling provides much better agreement with the frequencies of various modes, and then these processes are now regarded as being important even in the chemically normal stars.

- Inference of the solar internal rotation: If the sun would not be rotating, the eigenfrequencies of the modes with the same degree $l$ and the radial order $n$ would show the $(2 l+1)$-fold degeneracy. The degeneracy is lifted by rotation in reality. Analyses of this rotational splitting make it possible to determine the rotation profile in the sun as a function of the distance from the center and of the latitude. The rotation profile deduced from helioseismology is described more in detail in the next section.

- Finding of the "tachocline" layer: It was found that there is a shear layer just beneath the base of the convection zone, which is named the "tachocline" layer. A mixing process related with convective overshooting or rotational shear is likely to be induced. If the small scale turbulence in the thin layer is induced by shear instability, it will help to maintain the rigid rotation in the deeper zone.

- Finding of the "torsional oscillation" in the convection zone: Helioseismic investigation using p-modes as well as f-modes makes it possible to see the alternating zonal bands of slightly faster and slower rotation are superposed on the relatively smooth latitudinal variation in the rotational profile. These bands are so far found to reach to the depth of about $5 \%$ of the solar radius.

- Finding of the meridional motion in the convection zone: The latitudinal differential rotation would not be maintained without the transport of angular momentum from higher latitudes to lower latitudes or the other way around. Thus the presence of a meridional flow has long been expected. While the observational results about the meridional flow so far available are still limited to the horizontal motion in the meridional, the meridional flow thus obtained is poleward with a mean amplitude of $10 \mathrm{~m} \mathrm{~s}^{-1}$ at latitude $20^{\circ}$, and shows the peak-to-peak variation of $7 \mathrm{~m} \mathrm{~s}^{-1}$.

- Detection of solar-cycle variation in the frequencies and amplitudes of eigenmodes: A frequency change over the solar cycle has indeed been detected. However, it has not 


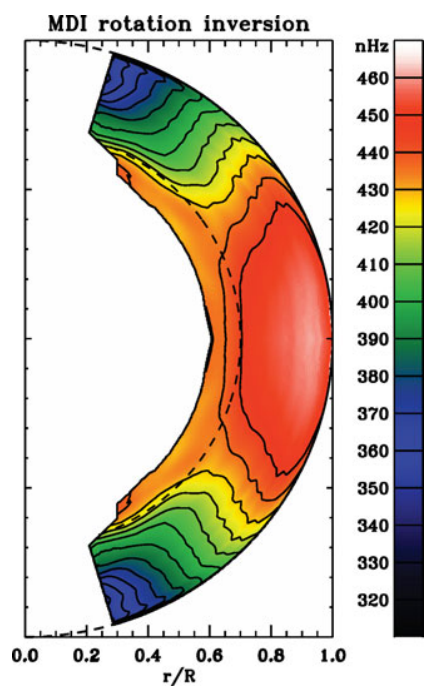

Figure 1. The rotation profile of $\Omega(r, \theta)$ in the solar envelope, inferred from helioseismic observations (Schou et al. 1998). The vertical axis is the rotation axis, and the dashed circle indicates the base of the convection zone. Contour labels are in $\mathrm{nHz}$. The white region indicates the region where reliable inference was not made at that time.

yet clarified the change in the thermal structure near the base of the convection zone which is believed to be the most plausible seat of the solar dynamo.

- Detection of solar-cycle variation in the solar internal dynamics: The bands of slowerand faster-than-average rotation migrating in latitude over the solar cycle in a manner similar to the zones of solar activity. The solar-cycle dependence of the meridional flow has also been investigated.

- Making it possible to look at the far-side of the sun and beneath the surface: Various techniques of local helioseismology have made it possible to infer thermal inhomogeneity and flows beneath the surface. Also active regions on the invisible far-side are now well inferred from helioseismic analyses of the visible side of the sun.

\section{Solar internal rotation}

The differential rotation is believed to be essential, with convective motion in the convection zone, for the solar dynamo mechanism, which must be responsible for the well-known 22-year solar activity cycle. The solar internal rotation is now observationally measurable from the $m$-splittings of the p-mode frequencies. Since the modes which penetrate into the very deep interior are limited to those of low degrees, and their splitting is smaller (being proportional to $2 l+1$ with the decrease of the degree $l$ ), it is hard to determine the internal rotation rate in the very deep interior. The same is true for the polar regions, since the modes which are sensitive to the polar regions are limited to low azimuthal order modes $(m \sim 0)$. Furthermore, since the sound speed becomes substantially higher with depth, p-modes are less sensitive to the deeper layers. This fact makes it harder to determine the internal rotation rate in the very deep interior. Hence, in figure 1, which is based on the analyses carried out about a decade ago, only the rotation profile of the accurately determinable region is shown. 
Conspicuous features are as follows:

- In the convection zone, the latitudinal differential rotation seen at the solar surface persists, more-or-less, even in the deeper layers. The rotational angular velocity in the convection zone is approximately a function of the latitude.

- The radiative core is rotating almost uniformly with a speed corresponding to the surface at mid-latitude - slower than the surface equatorial zone. According to the more recent observational results, the rotation rate seems to be approximately constant in the region between $r \simeq 0.2 R_{\odot}$ and the base of the convection zone.

- In the low-latitude zone, there is a radial gradient in the rotation profile in the convection zone, and the rotation rate is slower near the surface than in the slightly deeper layer .

- In the high-latitude zone, there is also a radial gradient in the rotation profile near the base of the convection zone, and the rotation rate becomes higher with approaching the bottom of the convection zone.

- The transition layer (the tachocline layer), in which the almost-latitudinal differential rotation turns to the almost-uniform rotation, is located just beneath the bottom of the convection zone, and it is very thin.

\section{Kinematic dynamo}

Let us consider the induction equation describing temporal variation of magnetic fields $\boldsymbol{B}$ in the MHD approximation:

$$
\frac{\partial \boldsymbol{B}}{\partial t}=\nabla \times(\boldsymbol{V} \times \boldsymbol{B})+\eta \nabla^{2} \boldsymbol{B}
$$

where $\boldsymbol{V}$ denotes the fluid velocity and $\eta$ is magnetic diffusivity. We consider the fluid motion in the solar convection zone and the magnetic fields there. At any given point and time, the physical variables of a system may be expressed in terms of mean values and fluctuating values. For such a decomposition to make sense, we assume that a suitable averaging spatial size is found so that the mean values are substantially independent of this averaging size. The fluid motion is then divided into the two parts, - the velocity field due to rotation of the sun, $\overline{\boldsymbol{V}}$, and that due to turbulent convection, $\boldsymbol{v} ; \boldsymbol{V}=\overline{\boldsymbol{V}}+\boldsymbol{v}$. Similarly, we divide the magnetic field into two components, - the mean field, $\overline{\boldsymbol{B}}$, and fluctuation, $\boldsymbol{b} ; \boldsymbol{B}=\overline{\boldsymbol{B}}+\boldsymbol{b}$.

We assume the influence of the magnetic field upon the mean motion is negligibly small, while the mean magnetic filed is generated and amplified by the presence of motion. This picture is called a kinematical dynamo. Such a model may be good enough to see where the magnetic field can be generated in the solar convection zone. With some appropriate assumptions (Parker 1955), the induction equation for the mean magnetic field is reduced to

$$
\frac{\partial \overline{\boldsymbol{B}}}{\partial t}=\nabla \times(\overline{\boldsymbol{V}} \times \overline{\boldsymbol{B}}+\alpha \overline{\boldsymbol{B}})+\left(\eta+\eta_{t}\right) \nabla^{2} \overline{\boldsymbol{B}}
$$

where $\alpha$ and $\eta_{t}$ are approximately given by

$$
\alpha \simeq-\frac{\tau}{3} \overline{\boldsymbol{v} \cdot \nabla \times \boldsymbol{v}}
$$

and

$$
\eta_{t} \simeq \frac{\tau}{3} \overline{\boldsymbol{v}^{2}}
$$

respectively, and $\tau$ is a representative lifetime of fluctuation. Note that the quantity 
$\overline{\boldsymbol{v} \cdot \nabla \times \boldsymbol{v}}$ is a measure of twist in convective motion and that it is positive in the case of clockwise twist with moving ahead.

Let us divide the mean magnetic field into the poloidal component $\overline{\boldsymbol{B}}_{\mathrm{p}}$ and the toroidal component $\bar{B}_{\phi}$, and consider these two components of equation (4.2). Also we hereafter ignore "bar" to express the mean field, as far as no confusion is expected. We further introduce the vector potential $A_{\phi}$ for the poloidal component:

$$
\boldsymbol{B}_{\mathrm{p}}=\nabla \times\left(A_{\phi} \mathbf{e}_{\phi}\right) \text {. }
$$

Then, equation (4.2) is reduced to the following two equations:

$$
\frac{\partial A_{\phi}}{\partial t}=-\frac{\boldsymbol{V}_{\mathrm{p}}}{r} \cdot \nabla\left(r A_{\phi}\right)+\eta\left(\nabla^{2}-\frac{1}{r^{2}}\right) A_{\phi}+\alpha B_{\phi}
$$

and

$$
\frac{\partial B_{\phi}}{\partial t}=r\left(\boldsymbol{B}_{\mathrm{p}} \cdot \nabla\right) \frac{V_{\phi}}{r}-r\left(\boldsymbol{V}_{\mathrm{p}} \cdot \nabla\right) \frac{B_{\phi}}{r}+\eta\left(\nabla^{2}-\frac{1}{r^{2}}\right) B_{\phi} .
$$

The last term in the RHS of equation (4.6) expresses the so-called $\alpha$-effect, by which the poloidal component of the magnetic field is generated by the toroidal component. The first term in the RHS of equation (4.7) means that the toroidal component is generated from the poloidal component with the help of rotation.

\section{Where does the dynamo work?}

It is instructive to consider the induction equations (4.6) and (4.7) for the mean magnetic field with the Cartesian coordinates and to treat them locally (Yoshimura 1975). Let us take the $z$-axis to point the vertical direction and let the $(x, y)$-plane be the horizontal plane at a given point. We take the $y$-direction eastward and the $x$-direction southward, respectively. Then, equations (4.6) and (4.7) are reduced to

$$
\left[\frac{\partial}{\partial t}-\eta \nabla^{2}\right] A_{y}=\alpha B_{y}
$$

and

$$
\left[\frac{\partial}{\partial t}-\eta \nabla^{2}\right] B_{y}=\frac{d V_{y}}{d z} \frac{\partial A_{y}}{\partial x}
$$

respectively.

In the local treatment, $d V_{y} / d z$ is treated as a constant, and $A_{y}$ and $B_{y}$ are assumed to be proportional to $\exp \left[i \omega t+i\left(k_{x} x+k_{z} z\right)\right]$. We take here $k_{x}>0$ without losing generality. Then, from equations (5.1) and (5.2), the following dispersion relation is derived:

$$
i \omega=\eta k^{2}\left[-1+(1 \pm i) \sqrt{\left|N_{\mathrm{D}}\right|}\right]
$$

where

$$
N_{\mathrm{D}} \equiv \frac{\alpha k_{x}}{2 \eta^{2} k^{4}} \frac{d V_{y}}{d z} .
$$

In the RHS of equation (5.3), the plus sign should be taken if $N_{\mathrm{D}}>0$ while the minus sign should be taken otherwise.

We know from the observed butterfly diagram, which shows the long-term variation in the latitude of the solar active regions, that the solar active region migrates equatorward (toward the direction of increase in $x$, in the northern hemisphere). Such a wave is 


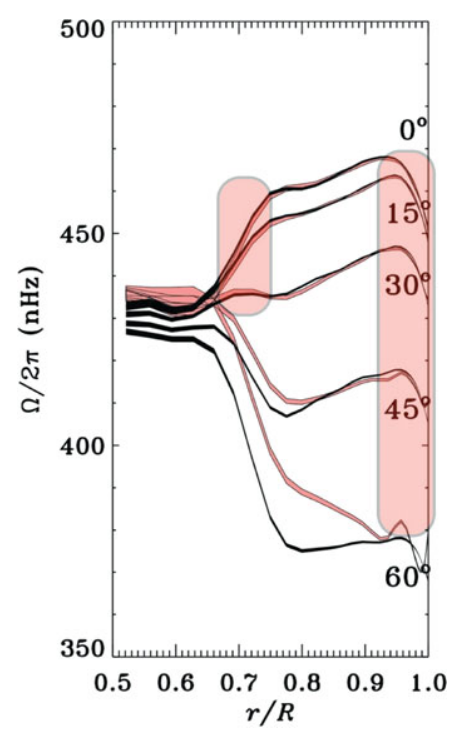

Figure 2. The rotation profiles of $\Omega(r, \theta)$ at various latitudes $\left(0^{\circ}, 15^{\circ}, 30^{\circ}, 45^{\circ}, 60^{\circ}\right)$ in the solar envelope, inferred from helioseismic observations. The hatched parts of the profiles satisfy the condition for the dynamo action.

expressed in terms of the dispersion relation with the minus sign in equation (5.3):

$$
i \omega=\eta k^{2}\left[-1+(1-i) \sqrt{\left|N_{\mathrm{D}}\right|}\right] .
$$

Hence $N_{\mathrm{D}}$ should be negative:

$$
N_{\mathrm{D}} \equiv \frac{\alpha k_{x}}{2 \eta^{2} k^{4}} \frac{d V_{y}}{d z}<0 .
$$

Note that the quantity $\alpha$ is related to kinetic helicity ( $\alpha$ has the opposite sign to kinetic helicity, $\overline{\boldsymbol{v} \cdot \nabla \times \boldsymbol{v}}$ ). Convective motion may be regarded as a plume moving upward from the bottom of the convection zone, where a fraction of gas elements converges horizontally to generate the plume, to the top layer, where the motion diverges horizontally. Coriolis force tends to twist counterclockwise the horizontally diverging flow near the top of the convection zone in the northern hemisphere while it twists clockwise the horizontally converging flow near the bottom of the convection zone. Hence $\alpha$ in the convection zone in the northern hemisphere is expected to be positive in the upper layer and negative in the lower layer. In this situation, the condition (5.6) requires $d V_{y} / d z<0$, which corresponds to $\partial \Omega / \partial r<0$ in the spherical coordinates, in the upper layer in the convective zone and $d V_{y} / d z>0$, which corresponds to $\partial \Omega / \partial r>0$, in the lower layer.

From this consideration, it is concluded that the dynamo action can work only near the upper layer of the convection zone at low and middle latitudes and/or near the low layer of the convective zone at low latitudes, as indicated in figure 2 .

\section{References}

Leighton, R. B., Noyes, R. W., \& Simon, G. W. 1962, ApJ, 135, 474

Parker, E. N. 1955, ApJ, 122, 293

Schou, J. et al. 1998, ApJ, 505, 390

Yoshimura, H. 1975, ApJ, 201, 740 